\title{
Reexamining the Solar Axion Explanation for the XENON1T Excess
}

\author{
Christina Gao $\oplus^{1, *}$ Jia Liu $\odot,{ }^{2,7, \dagger}$ Lian-Tao Wang, ${ }^{2,3, \$}$ Xiao-Ping Wang, ${ }^{4,8, \S}$ Wei Xue, ${ }^{5, \|}$ and Yi-Ming Zhong ${ }^{6, \|}$ \\ ${ }^{1}$ Theoretical Physics Department, Fermi National Accelerator Laboratory, Batavia, Illinois 60510, USA \\ ${ }^{2}$ Enrico Fermi Institute, University of Chicago, Chicago, Illinois 60637, USA \\ ${ }^{3}$ Department of Physics, University of Chicago, Chicago, Illinois 60637, USA \\ ${ }^{4}$ HEP Division, Argonne National Laboratory, 9700 Cass Ave., Argonne, Illinois 60439, USA \\ ${ }^{5}$ Department of Physics, University of Florida, Gainesville, Florida 32611, USA \\ ${ }^{6}$ Kavli Institute for Cosmological Physics, University of Chicago, Chicago, Illinois 60637, USA \\ ${ }^{7}$ School of Physics and State Key Laboratory of Nuclear Physics and Technology, Peking University, Beijing 100871, China \\ ${ }^{8}$ School of Physics, Beihang University, Beijing 100083, China
}

(Received 7 July 2020; accepted 28 August 2020; published 24 September 2020)

\begin{abstract}
The XENON1T collaboration has observed an excess in electronic recoil events below $5 \mathrm{keV}$ over the known background, which could originate from beyond-the-standard-model physics. The solar axion is a well-motivated model that has been proposed to explain the excess, though it has tension with astrophysical observations. The axions traveling from the Sun can be absorbed by the electrons in the xenon atoms via the axion-electron coupling. Meanwhile, they can also scatter with the atoms through the inverse Primakoff process via the axion-photon coupling, which emits a photon and mimics the electronic recoil signals. We found that the latter process cannot be neglected. After including the $\mathrm{keV}$ photon produced via the inverse Primakoff process in the detection, the tension with the astrophysical constraints can be significantly reduced. We also explore scenarios involving additional new physics to further alleviate the tension with the astrophysical bounds.
\end{abstract}

DOI: 10.1103/PhysRevLett.125.131806

Axions are pseudogoldstone bosons which naturally arise from the beyond-the-standard-model (BSM) physics scenarios [1-3]. Because of an approximate shift symmetry, they can be naturally light. Typically, they are very weakly coupled to other particles, which makes them a good candidate of dark matter or dark sector particles [4-6]. The phenomenology of the axions is rich, and they give unique signals in cosmology, astrophysics, and particle physics [7-11].

XENON1T, a dual-phase liquid xenon detector, is one of the leading experiments looking for dark matter (DM). Because of its large volume and low backgrounds, the XENON1T is also sensitive to other rare processes potentially related to BSM physics. Recently, the XENON1T collaboration reported their searches for the low-energy electronic recoil, with an excess in the range of $1-5 \mathrm{keV}$, which cannot be accounted for by the known backgrounds [12]. The XENON1T collaboration has also performed a fit to the excess using the solar axion model [13]. Since this report, there have been active speculations about the explanation of the excess [14-43].

Published by the American Physical Society under the terms of the Creative Commons Attribution 4.0 International license. Further distribution of this work must maintain attribution to the author(s) and the published article's title, journal citation, and DOI. Funded by SCOAP.
It is tempting to explain the XENON1T excess using the solar axions since the axion energy spectrum naturally matches the excess. The axions are produced in the Sun from several processes, including the Primakoff process $\gamma+Z e \rightarrow Z e+a$; the atomic axion-recombination and deexcitation, bremsstrahlung, and Compton (ABC) scattering processes; and the nuclear transitions. Hence, the axionphoton $g_{a \gamma}$, axion-electron $g_{a e}$, and axion-nucleon $g_{a n}$ couplings enter the production. With their tiny coupling to photons, the keVaxions have a long lifetime and can travel from the Sun to the XENON1T. For the processes in the detector which can give the signal, XENON1T [12] considered only the axion-electron coupling. In this case, the axions could be absorbed by the electrons in xenon atoms.

The relevant axion couplings can be summarized in the following Lagrangian:

$$
\mathcal{L} \supset-g_{a e} \frac{\partial_{\mu} a}{2 m_{e}} \bar{e} \gamma^{\mu} \gamma_{5} e-\frac{1}{4} g_{a \gamma} a F_{\mu \nu} \tilde{F}^{\mu \nu} .
$$

$F^{\mu \nu}$ is the field strength of the photon, and its dual $\tilde{F}^{\mu \nu}=\frac{1}{2} \epsilon^{\mu \nu \alpha \beta} F_{\alpha \beta}$. However, the parameter space of the solar axion interpretation of the excess is in tension with he astrophysical observations of stellar evolution including the white dwarfs (WDs) and the horizontal branch (HB) stars in the globular clusters (GCs) [12,25]. 


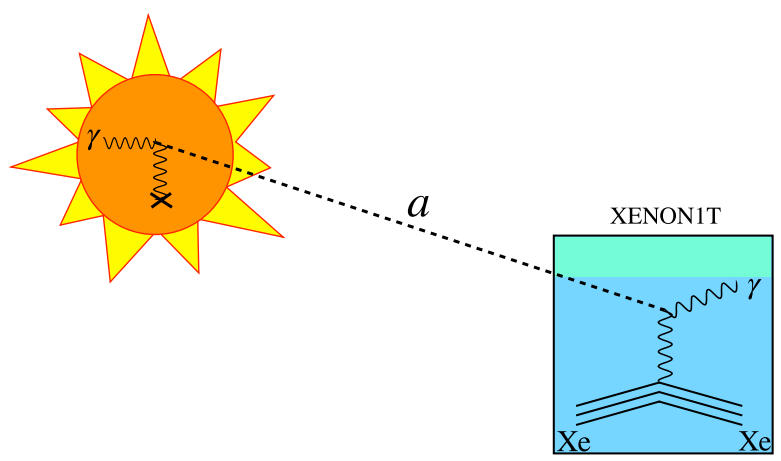

FIG. 1. The solar axion induced photon signal through the inverse Primakoff process.

In this Letter, we take into account the fact that, at the $\mathrm{keV}$ energy range, the current XENON1T experiment can hardly distinguish the detector response of photons from that of electronic recoils. Hence, instead of electronic recoil, the low-energy photons generated through the inverse Primakoff scattering between the solar axion and the xenon atoms in the detector can mimic the electronic signal, as shown in Fig. 1. Using the inverse Primakoff process to detect axions was proposed in the cryogenic experiments via Bragg scattering [44-46] and was applied by the SOLAX, COSME, CUORE, CDMS, and EDELWEISS collaborations [47-52]. However, it was not included in the liquid time projection chamber type of experiments previously. We show that, after including both the electronic recoil and the inverse Primakoff process, the tension between the solar axion explanation and the astrophysical constraints is significantly reduced.

The Letter is structured as follows: first, we describe the detection using the inverse Primakoff process, and after considering the astrophysics and terrestrial constraints, we present the fit to the data of XENON1T. Then, we discuss the possible extensions of new physics to further alleviate the tension between the constraints and the XENON1T fit. We conclude in the end.

Detection from the inverse Primakoff process. - In this section, we compute the contribution to the electronic recoil from the inverse Primakoff process $a+\mathrm{Xe} \rightarrow \gamma+\mathrm{Xe}$, where Xe represents the xenon nucleus. The differential cross section is given by $[44,46,53]$

$$
\frac{d \sigma_{a \rightarrow \gamma}^{\text {invPrim }}}{d \Omega}=\frac{\alpha}{16 \pi} g_{a \gamma}^{2} \frac{\boldsymbol{q}^{2}}{\boldsymbol{k}^{2}}\left(4-\boldsymbol{q}^{2} / \boldsymbol{k}^{2}\right) F_{a}^{2}\left(\boldsymbol{q}^{2}\right),
$$

where $\alpha$ is the fine structure constant, $\boldsymbol{k}$ is the momentum of the incoming axion and $\boldsymbol{q}$ is the momentum transfer. In the limit of small axion mass, $m_{a} \ll|\boldsymbol{k}|$, the energy of the outgoing photon is also approximately $|\boldsymbol{k}| . F_{a}$ is the form factor characterizing the screening effect of the electric charge of the nucleus, given by $F_{a}\left(\boldsymbol{q}^{2}\right)=Z \boldsymbol{k}^{2} /\left(r_{0}^{-2}+\boldsymbol{q}^{2}\right)$, where $Z=54$ is the atomic number of xenon and $r_{0}$ is the screening length [44], that can be determined numerically. We take the expression above and fit the form factor by the relation $F_{a}=\boldsymbol{k}^{2} / \boldsymbol{q}^{2}\left(Z-F_{\gamma}\right)$ [44], and the atomic form factor $F_{\gamma}$ is reported in Ref. [54]. The fit gives $r_{0}^{-1}=4.04 \mathrm{keV}=(49 \mathrm{pm})^{-1}$, which is close to the reciprocal of the xenon atomic radii $108 \mathrm{pm}$ [55]. This screening length corresponds to a screened charge of $Z_{s c}=5.3$ for xenon at $|\boldsymbol{q}|=3 \mathrm{keV}$.

Next, we calculate the event rate from solar axions with both the inverse Primakoff process and the axioelectric effect. The cross section of the latter process is given by $[56,57]$

$$
\sigma_{\mathrm{ae}}=\sigma_{\mathrm{pe}} \frac{g_{\mathrm{ae}}^{2}}{\beta_{a}} \frac{3 E_{a}^{2}}{16 \pi \alpha m_{e}^{2}}\left(1-\frac{\beta_{a}^{2 / 3}}{3}\right),
$$

where $\sigma_{\mathrm{pe}}$ is the photoelectric cross section [58] and $\beta_{a}$ is the axion velocity. We will focus on the low energy excess $(\lesssim 5 \mathrm{keV})$ throughout this Letter, hence, only consider the contributions to solar axion flux from the ABC process, $\Phi_{a}^{\mathrm{ABC}}$, and the Primakoff process, $\Phi_{a}^{\text {Prim }}$, and neglect that from the nuclear transition of ${ }^{57} \mathrm{Fe}$. The $\mathrm{ABC}$ flux originates from the axion-electron coupling and is given by $\Phi_{a}^{\mathrm{ABC}} \propto$ $g_{a e}^{2}$ [59]. The Primakoff flux is given by [60]

$$
\begin{aligned}
\frac{d \Phi_{a}^{\text {Prim }}}{d E_{a}}= & 6 \times 10^{10} \mathrm{~cm}^{-2} \mathrm{~s}^{-1} \mathrm{keV}^{-1} \\
& \times\left(\frac{g_{a \gamma}}{10^{-10} \mathrm{GeV}}\right)^{2}\left(\frac{E_{a}}{\mathrm{keV}}\right)^{2.481} e^{-E_{a} /(1.205 \mathrm{keV})} .
\end{aligned}
$$

Given the solar axion flux $\Phi_{a}$, the differential event rate after including both axioelectric and inverse Primakoff processes in the detection is given by

$$
\begin{aligned}
\frac{d R}{d E_{r}}= & \frac{N_{A}}{A}\left(\frac{d \Phi_{a}^{\mathrm{ABC}}}{d E}\left(E_{r}\right)+\frac{d \Phi_{a}^{\text {Prim }}}{d E}\left(E_{r}\right)\right) \\
& \times\left[\sigma_{a \rightarrow \gamma}^{\operatorname{invPrim}}\left(E_{r}\right)+\sigma_{a e}\left(E_{r}\right)\right],
\end{aligned}
$$

where $N_{A}$ is the Avogadro constant, $A=131$ is the atomic weight of xenon, and $E_{r}$ represents the electronic recoil energy, which is faked by photons in the inverse Primakoff process.

To compare with the results reported by the XENON1T collaboration, we further smear the differential event rate with a Gaussian with its variance satisfying $\sigma / E_{r}=$ $\left(a / \sqrt{E_{r}}+b\right) \%$. A numerical fit to the data of XENON1T energy resolution [61] yields $a=35.9929 \mathrm{keV}^{1 / 2}$ and $b=-0.2084$. After the smearing, we apply the detector efficiency [12].

Figure 2 shows two examples of the differential event rate of the electronic recoils given different values of $g_{a e}$ and $g_{a \gamma}$. In the case that $g_{a e}=0$, the spectrum is only determined by the detection of $\Phi_{a}^{\text {Prim }}$ through the inverse Primakoff process. It is clear that, with $g_{a e}$ switched off, solar axions can still account for the low energy excess, 

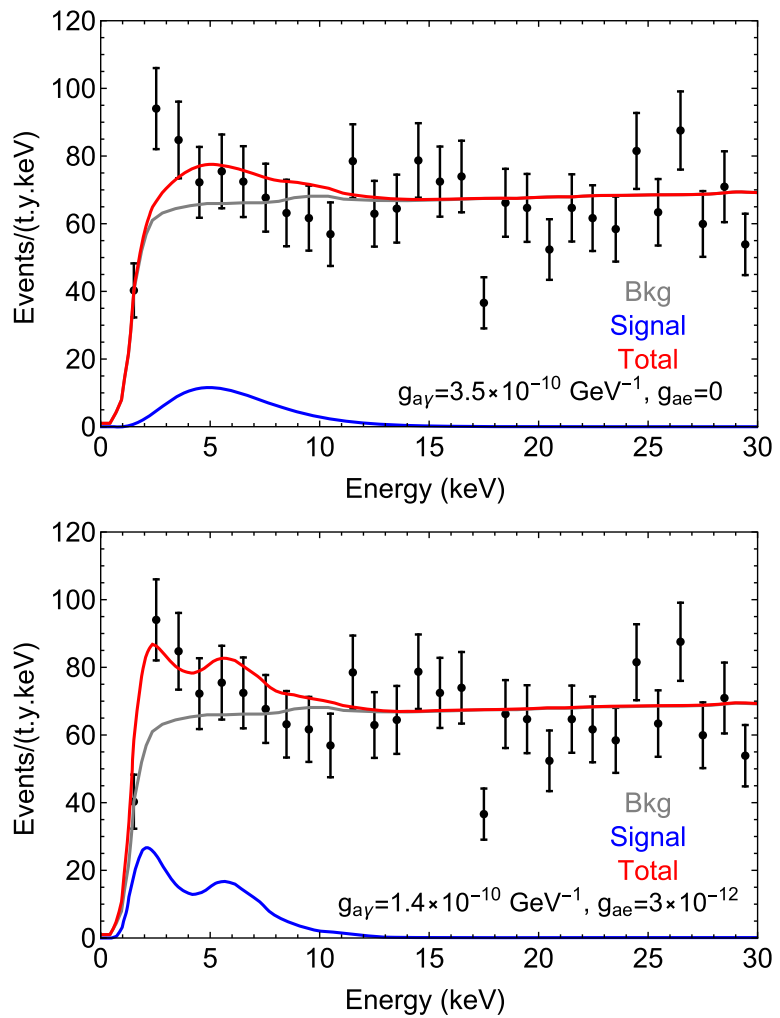

FIG. 2. Fit to electronic recoil energy spectrum with $g_{a \gamma}$ only (top) and both $g_{a \gamma}$ and $g_{a e}$ allowed (bottom).

although the fit is not as good as that allowing both $g_{a e}$ and $g_{a \gamma}$ to be nonzero.

Constraints from astrophysics and terrestrial experiments. - The most severe constraints on the solar axion explanation of the XENON1T excess are from the stellar cooling in the HB and red-giant branch (RGB) stars, which we review below.

Axions with sizable $g_{a \gamma}$ and $g_{a e}$ couplings speed up the burning of the He-core (H-core) for HB (RGB). The lifetime of the stars in the two phases is proportional to their observed numbers. Therefore, one can use the $R$ parameter, the ratio of the number of HB stars to that of RGB stars, to constrain the axion couplings. Reference [62] obtained a weighted average $R_{\mathrm{av}}=1.39 \pm 0.03$ from the $R$ parameters of 39 low-metallicity galactic GCs reported by [63]. Assuming $g_{a e}=0, g_{a \gamma}$ is constrained to be $g_{a \gamma}<$ $6.6 \times 10^{-11} \mathrm{GeV}^{-1}$. For nonzero $g_{a e}$, Ref. [64] presented two stellar evolution models which give slightly different predictions of the $R$ parameter. In Fig. 3, we adopted the resulting $95 \%$ C.L. constraints on the $g_{a e}-g_{a \gamma}$ plane for both models from Fig. 4 of [64]. In the Supplemental Material [65], we further discuss the bound dependence on the He abundance of GCs. The bremsstrahlung energy loss from the axion-electron coupling affects the white dwarf luminosity function (WDLF) and constrains $g_{a e} \lesssim 2.8 \times$ $10^{-13}$ [68]. The same argument on RGB constrains $g_{a e} \lesssim$ $4.3 \times 10^{-13}$ [69]. The global fit of the solar data constrains

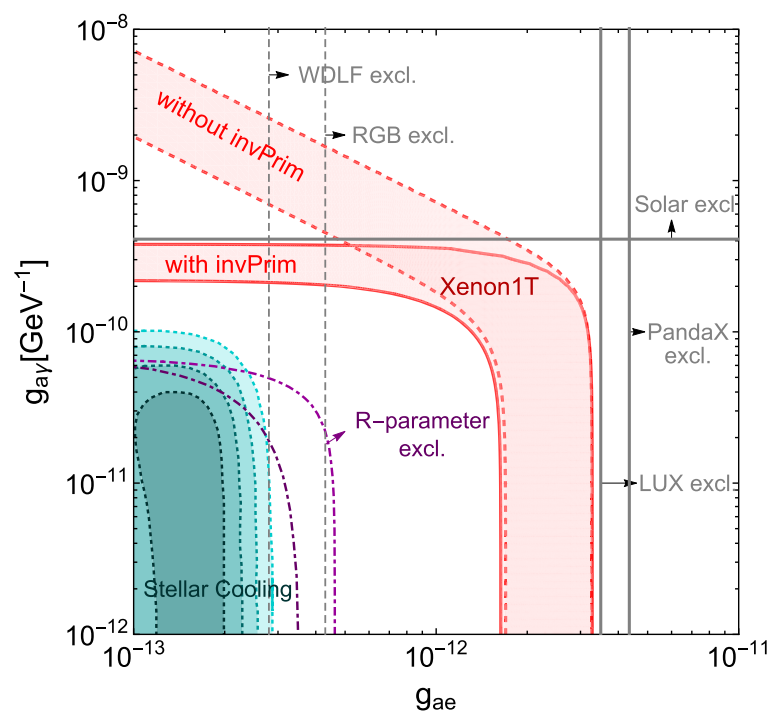

FIG. 3. he 2D axion couplings parameter fit for the XENON1T excess after including the inverse Primakoff process. Our best fit (90\% C.L.) to the XENON1T excess is shown in the red shaded region with the solid boundary. In comparison, a "XENON-like" analysis with only the electron recoil included as the signal yields is shown in the region with the dashed boundary. The main difference is that the inclusion of the inverse Primakoff process allows for a region in which $g_{a \gamma}$ is relatively large while $g_{a e}$ can be small, reducing the tension with the astrophysical data. Also included are the constraints (95\% C.L.) from astrophysical observables including WDLF [68], the tip of RGB [69] and the $R$ parameter (with two models) [64], as well as the constraints from the global fit of the solar data [70], LUX [73], and PandaX [74], with arrows denoting excluded regions. We do not show constraints from CAST [75] and magnetic WDs [71] by assuming the axion mass $\gtrsim 1 \mathrm{eV}$. The shaded green region contains $1 \sigma$ to $4 \sigma$ contours favored by the anomalous stellar cooling [25,72].

$g_{a \gamma}<4.1 \times 10^{-10} \mathrm{GeV}^{-1}$ [70]. Other constraints such as $\mathrm{x}$-ray observations on magnetic WDs [71] get significantly weakened for axion mass $\gtrsim 1 \mathrm{meV}$. In Fig. 3, we also show $1 \sigma$ to $4 \sigma g_{a e}-g_{a \gamma}$ contours favored by the anomalous stellar cooling $[25,72]$.

On the terrestrial experiments side, the axion searches from LUX [73] suggest $g_{\mathrm{ae}}<3.5 \times 10^{-12}$. Similar constraint is also shown by PandaX [74]. The CAST experiment [75] constrains light axions with $g_{a \gamma}<6.6 \times 10^{-11} \mathrm{GeV}^{-1}$. This bound gets significantly weakened for axions with mass $\gtrsim 1 \mathrm{eV}$.

Results.-In Fig. 3, we present our fit to the XENON1T excess and compare it with the bounds from the previous section. We scan two parameters $g_{a e}, g_{a \gamma}$, and apply the method of least squares to the XENON1T data to find the 90\% C.L. contours with (solid red) and without (dashed red) including the inverse Primakoff process. In comparison, we also show the constraints (95\% C.L.) from astrophysical observables including WDLF, the tip of RGB, and the $R$ parameter (with two models), as well as the constraints from the global fit of the solar data and 
the direct search at LUX and PandaX. The constraints from CAST and magnetic WDs can be evaded by the axion with a mass $\gtrsim 1 \mathrm{eV}$ and we do not show them in Fig. 3.

From Fig. 3, we see that the inclusion of the inversePrimakoff process has a significant impact on the parameter region preferred by the XENON1T data. In particular, it opens up a parameter region in which $g_{a \gamma} \gg g_{a e} / \mathrm{GeV}$ and the inverse Primakoff process gives rise to the observed signal. Moreover, for $g_{a e} \sim 10^{-13}$, it prefers a $g_{a \gamma}$ which is a few $\times 10^{-10} \mathrm{GeV}^{-1}, 1$ order of magnitude smaller than the preferred $g_{a \gamma}$ without the inclusion of the inverse Primakoff process, satisfying the constraints from the global fit of the solar data, and significantly reducing the tension with the stellar cooling bounds. Future terrestrial axion experiments, such as the International Axion Observatory [76] and the Multilayer Optical Haloscope [77], can complement the astrophysical probes and cover the relevant $g_{a \gamma}$ coupling region for axions with mass of several $\mathrm{eV}$.

Possible extensions. - Even though the inclusion of the inverse Primakoff process can significantly improve the prospect of explaining the XENON1T excess with the solar axions, it is still in tension with the stellar cooling bounds, with a discrepancy as large as $8 \sigma$, as claimed by [25]. Indeed, if the excess is completely due to new physics, there remains three possibilities. It could certainly come from other new physics instead of the solar axion, in which case a new explanation of the $\mathrm{keV}$ scale needs to be found. It is also possible that there is additional uncertainty in the stellar cooling bound which still has not been appreciated (see, e.g., [78,79] and the discussion in the Supplemental Material [65]). Instead of pursuing these avenues, we will explore a third possibility, with new physics in addition to the solar axion. In particular, we focus on the parameter space given by $g_{a \gamma} \gg g_{a e} / \mathrm{GeV}$, where the most relevant constraint is from the $R$ parameter, the cooling from HB. We introduce a $U(1)_{B}$ gauge boson and discuss its effects.

Consider an axion coupling to both photon and dark gauge boson $A^{\prime}$ carrying the $U(1)_{B}$ Baryon charge,

$$
\mathcal{L} \supset-\frac{1}{2} g_{a \gamma A^{\prime}} a F_{\mu \nu}^{\prime} \tilde{F}^{\mu \nu}+g_{B} A_{\mu}^{\prime} J_{\mathrm{B}}^{\mu} .
$$

The $U(1)_{B} A^{\prime}$ couples to the baryonic current $J_{B}$, but not directly to electrons. Hence, processes mediated by $A^{\prime}$ do not suffer the screening effect. Therefore, the Primakoff production in the Sun is increased if $A^{\prime}$ is lighter than the thermal photon in the plasma, and the detection cross section is enhanced by $A^{2} / Z_{s c}^{2} \sim 600$ for light $A^{\prime}$ with $m_{A^{\prime}}<r_{0}^{-1} \sim 4 \mathrm{keV}$.

The Primakoff cross section is given by

$\sigma_{\gamma \rightarrow a}^{A^{\prime} \text { Prim }}=\frac{g_{a \gamma A^{\prime}}^{2} \alpha_{B} A^{2}}{4}\left(\frac{2 \eta^{2}+1}{4 \eta^{2}} \ln \left(4 \eta^{2}+1\right)-1\right) F_{N}^{2}$,

where $\eta=|\boldsymbol{k}| / m_{A^{\prime}}, \boldsymbol{k}$ is the momentum of the axion, $\alpha_{B} \equiv g_{B}^{2} / 4 \pi$, and $F_{N}$ is the nuclear form factor which is approximately 1 for a momentum transfer of a few $\mathrm{keV}$. We follow Ref. [53] to calculate the Primakoff energy loss due to Eq. (6) for $A^{\prime}$ with $m_{A^{\prime}}=0.1(1) \mathrm{keV}$. The resulting solar energy loss rate per unit volume is

$Q_{a}^{A^{\prime} \operatorname{Prim}}(\operatorname{Sun}) \approx Q_{a}^{\text {Prim }}(\operatorname{Sun}) \times 16.9(4.3) \times \frac{\alpha_{B}}{\alpha} \frac{g_{a \gamma A^{\prime}}^{2}}{g_{a \gamma}^{2}}$,

and that for $\mathrm{HB}$ is

$Q_{a}^{A^{\prime} \operatorname{Prim}}(\mathrm{HB})=Q_{a}^{\mathrm{Prim}}(\mathrm{HB}) \times 15.6(8.0) \times \frac{\alpha_{B}}{\alpha} \frac{g_{a \gamma A^{\prime}}^{2}}{g_{a \gamma}^{2}}$,

where $Q_{a}^{\text {Prim }}$ is the energy loss rate per unit volume from Eq. (1). The cross section for inverse Primakoff detection at XENON1T is given by $\sigma_{a \rightarrow \gamma}^{A^{\prime} \text { invPrim }}=2 \sigma_{\gamma \rightarrow a}^{A^{\prime} \text { Prim }}$. For $m_{A^{\prime}}=0.1(1) \mathrm{keV}$,

$$
\sigma_{a \rightarrow \gamma}^{A^{\prime} \text { invPrim }}=\sigma_{a \rightarrow \gamma}^{\text {invPrim }} \times 400(90) \times \frac{\alpha_{B}}{\alpha} \frac{g_{a \gamma A^{\prime}}^{2}}{g_{a \gamma}^{2}} .
$$

Combining the solar axion flux and the detection cross section, we find that for $m_{A^{\prime}}=0.1(1) \mathrm{keV}$, it requires

$$
g_{a \gamma A^{\prime}} g_{B} \approx 0.11(0.23) g_{a \gamma} e,
$$

to explain the XENON1T excess. Moreover, this choice of parameter helps to alleviate the HB cooling tension, such that its energy loss is reduced to $19 \%(40 \%)$ of that solely due to axion from Eq. (1).

However, there are severe constraints for $U(1)_{B}$ couplings from astrophysics and collider physics. The stars (the Sun, HB, and supernova) can be cooled by directly emitting $A^{\prime}$ through bremsstrahlung and Compton scattering. The constraint from SN1987 A for $U(1)_{B}$ is $g_{B} \lesssim$ $2.5 \times 10^{-10}[80]$. For solar and HB cooling, the emission of $A^{\prime}$ from the ion leg is suppressed by $\mathcal{O}\left(m_{e}^{2} / m_{n}^{2}\right)$ and, thus, requires $g_{B} \lesssim 10^{-10}$ [81,82]. For collider physics, the UV anomaly cancellation of $U(1)_{B}$ leads to Wess-Zumino operators at low energy, which constrains $g_{B} / m_{A^{\prime}}<3 \times$ $10^{-10} \mathrm{keV}^{-1}$ [83] from the invisible decays of the $Z$ boson or mesons. Therefore, both sets of constraints suggest that $g_{B} \lesssim 10^{-10}$ for $\mathrm{keV} A^{\prime}$.

To explain the XENON1T excess, the coupling $g_{a \gamma A^{\prime}}$ should be larger than $\sim 0.1 \mathrm{GeV}^{-1}$, meaning a cutoff scale of $10 \mathrm{GeV}$. Such an energy scale may arise from integrating out new light particles [31]. However, the thermal photon in the plasma can decay via $\gamma^{*} \rightarrow a+A^{\prime}$, thus, a large $g_{a \gamma A^{\prime}}$ is not desirable. In summary, both $g_{B}$ and $g_{a \gamma A^{\prime}}$ are highly constrained, such that they can not account for the excess. One might also consider large $g_{B}$ to evade the cooling bounds, because the emitted $A^{\prime}$ is then trapped inside the star. Using a simple mean-free-path criterion, $\left(n_{I} \sigma_{A^{\prime} I \rightarrow \gamma I}\right)^{-1} \lesssim r_{\text {star }}$, where $\sigma_{A^{\prime} I \rightarrow \gamma I}$ stands for the Compton scattering, the trapping is realized with $g_{B} \gtrsim 10^{-2}$ for the Sun and HB. $A^{\prime}$ with a large $g_{B}$ can have a sizable coupling 
to electrons via one-loop induced kinetic mixing, which is constrained by the $(g-2)_{e}$ measurement to be $g_{e} \lesssim 10^{-5}$ [84]. The loop induced coupling to electron is on the order of $g_{e} \sim g_{B} \alpha$. However, there can also be a UV contribution to the kinetic mixing as well. With fine tuning, the total coupling to the electron can be made to be consistent with the constraint. The resulting scenario could have interesting implications which deserve further study.

We also consider using the environment effect to weaken the HB cooling bound while keeping the solar axion flux intact. The core temperature of $\mathrm{HB}$ and the Sun are 10 and $1 \mathrm{keV}$, and the corresponding photon plasma mass $\omega_{p}$ are 2 and $0.3 \mathrm{keV}$, respectively. Consider axions coupling to both photon and $U(1)^{\prime}$ dark photon,

$$
\mathcal{L} \supset-\frac{1}{4} g_{a \gamma} a F_{\mu \nu} \tilde{F}^{\mu \nu}-\frac{1}{2} g_{a \gamma A^{\prime}} a F_{\mu \nu}^{\prime} \tilde{F}^{\mu \nu} .
$$

The resulting Primakoff production of axions contains diagrams with $t$-channel $\gamma$ or $A^{\prime}$. One can choose the sign of $g_{a \gamma A^{\prime}}$ to have destructive interference among the two diagrams and make them cancel for $\mathrm{HB}$ with a particular combination of $g_{a \gamma A^{\prime}} g_{B}$. However, a complete cancellation only works for particular momentum transfer and, thus, does not apply to the entire phase space. In addition, the core temperature for $\mathrm{HB}$ is not a constant, thus, the cancellation may only happen within a restricted volume. Therefore, it is difficult to have a significant environmentdependent suppression.

Above, we focused on the solar axions, where the origin of the keV scale of the excess can be naturally explained. Nevertheless, it is hard to accommodate the stellar cooling bounds. Dropping the relation between the $\mathrm{keV}$ scale and the solar axion energy, one may consider a broader range of the signal sources. For example, the $U(1)_{B}$ dark photon could be the dark matter, with mass $m_{A^{\prime}} \simeq 2.8 \mathrm{keV}$. In this case, the detection at XENON1T is through the dark photon conversion $A^{\prime}+\mathrm{Xe} \rightarrow \gamma+\mathrm{Xe}$ with the cross-section given by

$$
\sigma v=A^{2} Z_{s c}^{2} \frac{e^{2} g_{B}^{2}}{6 \pi m_{\mathrm{Xe}}^{2}},
$$

where $A=131, Z_{s c} \sim 5.3$. Since $A^{\prime}$ is much lighter than the nucleus, the photon energy is about $m_{A^{\prime}}$. The total number of events is given by (exposure $\left./ m_{\mathrm{Xe}}\right) \sigma v\left(\rho_{\mathrm{DM}} / m_{A^{\prime}}\right)$, where $\rho_{\text {DM }}=0.3 \mathrm{GeV} / \mathrm{cm}^{3}$ is the local DM density. The excess can be explained with $g_{B} \approx 5 \times 10^{-11}$. Such a value is marginally below that from the astrophysical and collider bounds $g_{B} \lesssim 10^{-10}$. References [12,42] studied kinetic mixing dark photon dark matter as a solution for the excess and requires the mixing parameter $\epsilon \approx 7 \times 10^{-16}$. The difference between those models and ours comes from the dark photon coupling to electrons. The resulting dark-photoelectric cross section is approximately $\left(m_{N}^{2} / m_{e}^{2}\right)\left(\epsilon^{2} / g_{B}^{2}\right)$ times of Eq. (13). Though there is a large difference in the value of couplings, both scenarios are marginally allowed given the astrophysical constraints.

Conclusions. - Solar axion is an appealing explanation for the XENON1T excess, with its energy naturally in the $\mathrm{keV}$ range. In this Letter, we have emphasized the importance of including photons with a similar recoil spectrum as a possible explanation for the XENON1T excess. In particular, it can significantly reduce the tension between the solar axion explanation and the astrophysical data, especially the stellar cooling bound. Introducing additional new physics can further alleviate the remaining tension.

We conclude, here, by briefly discussing future prospects. We expect that further sharpening the stellar cooling bound will certainly help to clarify the situation. Indeed, if there is additional new physics that helps to relieve the tension with the astrophysical bound, it would be interesting in exploring other possible signals of these new physics. For example, a more sensitive search for the $U(1)_{B}$ can have the potential of shedding new light on this scenario. We also note that it is possible to have new physics models in which the photon comes from completely different sources. For example, it can come from a different dark matter scattering process [31] or from decaying from an excited state of the dark matter [26,41], or the dark photon conversion process considered in this Letter. In these cases, the spectrum of the photon would be different from the one from the inverse Primakoff process. Future data can be used to distinguish these scenarios.

We would like thank Luca Grandi, Tarek Saab, Evan Shockley for discussing in detail the response to the electron and photon in the XENON1T detector, Fei Gao, Jingqiang Ye for the details of fit and the analysis, Aaron Pierce and Sergey Sibiryakov for the axion models and constraints, and Maurizio Giannotti, Andreas Ringwald, and Oscar Straniero for clarifying the weighted average for the $R$ parameter and the theoretical prediction formula. C. G. is supported by Fermi Research Alliance, LLC under Contract No. DE-AC02-07CH11359, J. L. acknowledges support by an Oehme Fellowship, L. T. W. is supported by the DOE Grant No. DE-SC0013642, X. P. W. is supported by the DOE Grant No. DE-AC02-06CH11357, W. X. is supported by the DOE Grant No. DE-SC0010296, and Y.Z. is supported by the Kavli Institute for Cosmological Physics at the University of Chicago through an endowment from the Kavli Foundation and its founder Fred Kavli. J. L. and X.P.W. have moved to Peking University and Beihang University respectively, during the review and revision processes.

Note added.-Shortly after this work appeared on arXiv, a study [85] appeared that also investigated the inverse Primakoff effect on the solar axion detection.

\footnotetext{
*Corresponding author. yanggao@fnal.gov Corresponding author. liuj1@uchicago.edu
} 
${ }^{\ddagger}$ Corresponding author.

liantaow@uchicago.edu

${ }^{\S}$ Corresponding author.

xia.wang@anl.gov

Corresponding author.

weixue@ufl.edu

Corresponding author.

ymzhong@kicp.uchicago.edu

[1] R. D. Peccei and H. R. Quinn, $C P$ Conservation in the Presence of Instantons, Phys. Rev. Lett. 38, 1440 (1977).

[2] S. Weinberg, A New Light Boson?, Phys. Rev. Lett. 40, 223 (1978).

[3] F. Wilczek, Problem of Strong $P$ and $T$ Invariance in the Presence of Instantons, Phys. Rev. Lett. 40, 279 (1978)

[4] J. Preskill, M. B. Wise, and F. Wilczek, Cosmology of the invisible axion, Phys. Lett. 120B, 127 (1983).

[5] L. F. Abbott and P. Sikivie, A cosmological bound on the invisible axion, Phys. Lett. 120B, 133 (1983).

[6] M. Dine and W. Fischler, The not so harmless axion, Phys. Lett. 120B, 137 (1983).

[7] G. G. Raffelt, Astrophysical methods to constrain axions and other novel particle phenomena, Phys. Rep. 198, 1 (1990).

[8] L. D. Duffy and K. van Bibber, Axions as dark matter particles, New J. Phys. 11, 105008 (2009).

[9] M. Kawasaki and K. Nakayama, Axions: Theory and cosmological role, Annu. Rev. Nucl. Part. Sci. 63, 69 (2013).

[10] D. J.E. Marsh, Axion cosmology, Phys. Rep. 643, 1 (2016).

[11] P. W. Graham, I. G. Irastorza, S. K. Lamoreaux, A. Lindner, and K. A. van Bibber, Experimental searches for the axion and axion-like particles, Annu. Rev. Nucl. Part. Sci. 65, 485 (2015).

[12] E. Aprile et al. (XENON Collaboration), Observation of excess electronic recoil events in XENON1T, arXiv: 2006.09721.

[13] K. van Bibber, P. M. McIntyre, D. E. Morris, and G. G. Raffelt, A practical laboratory detector for solar axions, Phys. Rev. D 39, 2089 (1989).

[14] F. Takahashi, M. Yamada, and W. Yin, XENON1T anomaly from anomaly-free ALP dark matter and its implications for stellar cooling anomaly, arXiv:2006.10035.

[15] C. A. O'Hare, A. Caputo, A. J. Millar, and E. Vitagliano, Axion helioscopes as solar magnetometers, Phys. Rev. D 102, 043019 (2020).

[16] K. Kannike, M. Raidal, H. Veermäe, A. Strumia, and D. Teresi, Dark matter and the XENON1T electron recoil excess, arXiv:2006.10735.

[17] D. W. P. do Amaral, D. G. Cerdeno, P. Foldenauer, and E. Reid, Solar neutrino probes of the muon anomalous magnetic moment in the gauged $U(1)_{L_{\mu}-L_{\tau}}$, arXiv:2006.11225.

[18] G. Alonso-Álvarez, F. Ertas, J. Jaeckel, F. Kahlhoefer, and L. Thormaehlen, Hidden photon dark matter in the light of XENON1T and stellar cooling, arXiv:2006.11243.

[19] B. Fornal, P. Sandick, J. Shu, M. Su, and Y. Zhao, Boosted dark matter interpretation of the XENON1T excess, arXiv:2006.11264.
[20] C. Boehm, D. G. Cerdeno, M. Fairbairn, P. A. Machado, and A. C. Vincent, Light new physics in XENON1T, arXiv: 2006.11250.

[21] K. Harigaya, Y. Nakai, and M. Suzuki, Inelastic dark matter electron scattering and the XENON1T excess, arXiv: 2006.11938.

[22] A. Bally, S. Jana, and A. Trautner, Neutrino self-interactions and XENON1T electron recoil excess, arXiv:2006.11919.

[23] L. Su, W. Wang, L. Wu, J. M. Yang, and B. Zhu, Xenon1T anomaly: Inelastic cosmic ray boosted dark matter, arXiv: 2006.11837.

[24] M. Du, J. Liang, Z. Liu, V. Q. Tran, and Y. Xue, On-shell mediator dark matter models and the Xenon1T anomaly, arXiv:2006.11949.

[25] L. Di Luzio, M. Fedele, M. Giannotti, F. Mescia, and E. Nardi, this issue, Solar Axions Cannot Explain the XENON1T Excess, Phys. Rev. Lett. 125, 131804 (2020).

[26] N. F. Bell, J. B. Dent, B. Dutta, S. Ghosh, J. Kumar, and J. L. Newstead, Explaining the XENON1T excess with luminous dark matter, arXiv:2006.12461.

[27] Y. Chen, J. Shu, X. Xue, G. Yuan, and Q. Yuan, Sun heated MeV-scale dark matter and the XENON1T electron recoil excess, arXiv:2006.12447.

[28] D. Aristizabal Sierra, V. De Romeri, L. Flores, and D. Papoulias, Light vector mediators facing XENON1T data, Phys. Lett. B 809, 135681 (2020).

[29] J. Buch, M. A. Buen-Abad, J. Fan, and J. S. C. Leung, Galactic origin of relativistic bosons and XENON1T excess, arXiv:2006.12488.

[30] G. Choi, M. Suzuki, and T. T. Yanagida, XENON1T anomaly and its implication for decaying warm dark matter, arXiv:2006.12348.

[31] G. Paz, A. A. Petrov, M. Tammaro, and J. Zupan, Shining dark matter in Xenon1T, arXiv:2006.12462.

[32] U. K. Dey, T. N. Maity, and T. S. Ray, Prospects of Migdal effect in the explanation of XENON1T electron recoil excess, arXiv:2006.12529.

[33] A. N. Khan, Can nonstandard neutrino interactions explain the XENON1T spectral excess?, arXiv:2006.12887.

[34] Q.-H. Cao, R. Ding, and Q.-F. Xiang, Exploring for sub$\mathrm{MeV}$ boosted dark matter from Xenon electron direct detection, arXiv:2006.12767.

[35] R. Primulando, J. Julio, and P. Uttayarat, Collider Constraints on a dark matter interpretation of the XENON1T excess, arXiv:2006.13161.

[36] K. Nakayama and Y. Tang, Gravitational production of hidden photon dark matter in light of the XENON1T excess, arXiv:2006.13159.

[37] K. Van Tilburg, Stellar basins of gravitationally bound particles, arXiv:2006.12431.

[38] H. M. Lee, Exothermic dark matter for XENON1T excess, arXiv:2006.13183.

[39] G. B. Gelmini, V. Takhistov, and E. Vitagliano, Scalar direct detection: In-medium effects, arXiv:2006.13909.

[40] Y. Jho, J.-C. Park, S. C. Park, and P.-Y. Tseng, Gauged lepton number and cosmic-ray boosted dark matter for the XENON1T excess, arXiv:2006.13910.

[41] M. Baryakhtar, A. Berlin, H. Liu, and N. Weiner, Electromagnetic signals of inelastic dark matter scattering, arXiv:2006.13918. 
[42] H. An, M. Pospelov, J. Pradler, and A. Ritz, New limits on dark photons from solar emission and keV scale dark matter, arXiv:2006.13929.

[43] J. Smirnov and J. F. Beacom, New Freezeout Mechanism for Strongly Interacting Dark Matter, Phys. Rev. Lett. 125, 131301 (2020).

[44] W. Buchmuller and F. Hoogeveen, Coherent production of light scalar particles in Bragg scattering, Phys. Lett. B 237, 278 (1990).

[45] E. A. Paschos and K. Zioutas, A proposal for solar axion detection via Bragg scattering, Phys. Lett. B 323, 367 (1994).

[46] R. J. Creswick, F. T. Avignone III, H. A. Farach, J. I. Collar, A. O. Gattone, S. Nussinov, and K. Zioutas, Theory for the direct detection of solar axions by coherent Primakoff conversion in germanium detectors, Phys. Lett. B 427, 235 (1998).

[47] F. T. Avignone III et al. (SOLAX Collaboration), Experimental Search for Solar Axions via Coherent Primakoff Conversion in a Germanium Spectrometer, Phys. Rev. Lett. 81, 5068 (1998).

[48] A. Morales et al. (COSME Collaboration), Particle dark matter and solar axion searches with a small germanium detector at the Canfranc underground laboratory, Astropart. Phys. 16, 325 (2002).

[49] C. Arnaboldi et al. (CUORE Collaboration), CUORE: A cryogenic underground observatory for rare events, Nucl. Instrum. Methods Phys. Res., Sect. A 518, 775 (2004).

[50] C. Arnaboldi et al. (CUORE Collaboration), Physics potential and prospects for the CUORICINO and CUORE experiments, Astropart. Phys. 20, 91 (2003).

[51] Z. Ahmed et al. (CDMS Collaboration), Search for Axions with the CDMS Experiment, Phys. Rev. Lett. 103, 141802 (2009).

[52] E. Armengaud et al., Axion searches with the EDELWEISSII experiment, J. Cosmol. Astropart. Phys. 11 (2013) 067.

[53] G. Raffelt, Stars as Laboratories for Fundamental Physics: The Astrophysics of Neutrinos, Axions, and Other Weakly Interacting Particles (University of Chicago Press, Chicago, 1996).

[54] P. J. Brown, A. G. Fox, E. N. Maslen, M. A. O'Keefe, and B.T.M. Willis, Intensity of diffracted intensities, International Tables for Crystallography (WILEY, Hoboken, 2006), Chap. 6.1, p. 554.

[55] E. Clementi, D. L. Raimondi, and W. P. Reinhardt, Atomic screening constants from scf functions. II. Atoms with 37 to 86 electrons, J. Chem. Phys. 47, 1300 (1967).

[56] M. Pospelov, A. Ritz, and M. B. Voloshin, Bosonic superWIMPs as keV-scale dark matter, Phys. Rev. D 78, 115012 (2008).

[57] F. Alessandria et al. (CUORE Collaboration), Search for $14.4 \mathrm{keV}$ solar axions from M1 transition of Fe-57 with CUORE crystals, J. Cosmol. Astropart. Phys. 05 (2013) 007.

[58] K. Arisaka, P. Beltrame, C. Ghag, J. Kaidi, K. Lung, A. Lyashenko, R. D. Peccei, P. Smith, and K. Ye, Expected sensitivity to Galactic/Solar axions and bosonic super-WIMPs based on the axio-electric effect in liquid Xenon dark matter detectors, Astropart. Phys. 44, 59 (2013).

[59] J. Redondo, Solar axion flux from the axion-electron coupling, J. Cosmol. Astropart. Phys. 12 (2013) 008.

[60] B. B. M. Kuster and G. Raffelt, Axions (Springer-Verlag, Berlin, Heidelberg, 2008).

[61] E. Aprile et al. (XENON Collaboration), Observation of two-neutrino double electron capture in ${ }^{124} \mathrm{Xe}$ with XENON1T, Nature (London) 568, 532 (2019).

[62] A. Ayala, I. Domínguez, M. Giannotti, A. Mirizzi, and O. Straniero, Revisiting the Bound on Axion-Photon Coupling from Globular Clusters, Phys. Rev. Lett. 113, 191302 (2014).

[63] M. Salaris, M. Riello, S. Cassisi, and G. Piotto, The initial helium abundance of the Galactic globular cluster system, Astron. Astrophys. 420, 911 (2004).

[64] M. Giannotti, I. Irastorza, J. Redondo, and A. Ringwald, Cool WISPs for stellar cooling excesses, J. Cosmol. Astropart. Phys. 05 (2016) 057.

[65] See Supplemental Material at http://link.aps.org/ supplemental/10.1103/PhysRevLett.125.131806 for the bound dependence on the $\mathrm{He}$ abundance of GCs, which includes Refs. [62,64,66,67].

[66] M. Tanabashi et al. (Particle Data Group), Review of particle physics, Phys. Rev. D 98, 030001 (2018).

[67] A. M. Serenelli and S. Basu, Determining the initial helium abundance of the sun, Astrophys. J. 719, 865 (2010).

[68] M. M. M. Bertolami, B. E. Melendez, L. G. Althaus, and J. Isern, Revisiting the axion bounds from the Galactic white dwarf luminosity function, J. Cosmol. Astropart. Phys. 10 (2014) 069.

[69] N. Viaux, M. Catelan, P. B. Stetson, G. G. Raffelt, J. Redondo, A. A. R. Valcarce, and A. Weiss, Neutrino and Axion Bounds from the Globular Cluster M5 (NGC 5904), Phys. Rev. Lett. 111, 231301 (2013).

[70] N. Vinyoles, A. Serenelli, F. L. Villante, S. Basu, J. Redondo, and J. Isern, New axion and hidden photon constraints from a solar data global fit, J. Cosmol. Astropart. Phys. 10 (2015) 015.

[71] C. Dessert, A. J. Long, and B. R. Safdi, X-Ray Signatures of Axion conversion in Magnetic White Dwarf Stars, Phys. Rev. Lett. 123, 061104 (2019).

[72] M. Giannotti, I. G. Irastorza, J. Redondo, A. Ringwald, and K. Saikawa, Stellar recipes for axion hunters, J. Cosmol. Astropart. Phys. 10 (2017) 010.

[73] D. S. Akerib et al. (LUX Collaboration), First Searches for Axions and Axionlike Particles with the LUX Experiment, Phys. Rev. Lett. 118, 261301 (2017).

[74] C. Fu et al. (PandaX Collaboration), Limits on Axion Couplings from the First 80 Days of Data of the PandaX-II Experiment, Phys. Rev. Lett. 119, 181806 (2017).

[75] V. Anastassopoulos et al. (CAST Collaboration), New CAST limit on the axion-photon interaction, Nat. Phys. 13, 584 (2017).

[76] E. Armengaud et al., Conceptual design of the International Axion Observatory (IAXO), J. Instrum. 9, T05002 (2014). 
[77] M. Baryakhtar, J. Huang, and R. Lasenby, Axion and hidden photon dark matter detection with multilayer optical haloscopes, Phys. Rev. D 98, 035006 (2018).

[78] G. G. Raffelt, Bound on neutrino dipole moments from globular clusters, J. Phys. G 17, S269 (1991).

[79] R. G. Gratton, E. Carretta, A. Bragaglia, S. Lucatello, and V. D'Orazi, The second and third parameters of the horizontal branch in globular clusters, Astron. Astrophys. 517, A81 (2010).

[80] E. Rrapaj and S. Reddy, Nucleon-nucleon bremsstrahlung of dark gauge bosons and revised supernova constraints, Phys. Rev. C 94, 045805 (2016).

[81] R. Harnik, J. Kopp, and P. A. N. Machado, Exploring nu signals in dark matter detectors, J. Cosmol. Astropart. Phys. 07 (2012) 026.
[82] E. Hardy and R. Lasenby, Stellar cooling bounds on new light particles: plasma mixing effects, J. High Energy Phys. 02 (2017) 033.

[83] J. A. Dror, R. Lasenby, and M. Pospelov, Dark forces coupled to nonconserved currents, Phys. Rev. D 96, 075036 (2017).

[84] S. Knapen, T. Lin, and K. M. Zurek, Light dark matter: Models and constraints, Phys. Rev. D 96, 115021 (2017).

[85] J. B. Dent, B. Dutta, J. L. Newstead, and A. Thompson, preceding Letter, Inverse Primakoff Scattering as a Probe of Solar Axions at Liquid Xenon Direct Detection Experiments, Phys. Rev. Lett. 125, 131805 (2020). 